\title{
Modeling Non-aqueous Phase Liquid Displacement Process
}

\author{
Yang Zhenqing, Shao Changjin, Zhou Guanggang and Qiu Chao \\ (Physics Department, China University of Petroleum, Beijing 102249, China)
}

\begin{abstract}
A pore-network model physically based on pore level multiphase flow was used to study the water-non-aqueous phase liquid (NAPL) displacement process, especially the effects of wettability, water-NAPL interfacial tension, the fraction of NAPL-wet pores, and initial water saturation on the displacement. The computed data show that with the wettability of the mineral surfaces changing from strongly water-wet to NAPL-wet, capillary pressure and the NAPL relative permeability gradually decrease, while water-NAPL interfacial tension has little effect on water relative permeability, but initial water saturation has a strong effect on water and NAPL relative permeabilities. The analytical results may help to understand the micro-structure displacement process of non-aqueous phase liquid and to provide the theoretical basis for controlling NAPL migration.
\end{abstract}

Key words: Non-aqueous phase liquid, pore-network model, capillary pressure, relative permeability

\section{Introduction}

Non-aqueous phase liquids (NAPLs) are water-immiscible hydrocarbons in the subsurface that exhibit behavior and properties different from dissolved contaminants. While dissolved contaminants are invisible to the naked eye and migrate with the flow of groundwater, NAPL forms a visible, separate oily phase in the subsurface and its migration is governed by gravity, buoyancy, and capillary forces (Bedient, et al., 1994). When being released at the surface, free-phase or mobile NAPL is forced into the pores of the soil/aquifer matrix by the hydrostatic pressure on the continuous body of NAPL. Because NAPL is under pressure, it can enter even very small pores and fractures in the subsurface as long as the original NAPL entry point, such as a waste pond or leaking underground storage tank, is active. When the supply of new NAPL is exhausted, however, the pressure on the free-phase NAPL is removed and small blobs of NAPL "snap-off" or "bypass" the once continuous NAPL body and become trapped in individual pores or small groups of pores by capillary force.

Any prediction of temporal and spatial distribution of these fluids is sensitive to the macroscopic descriptors of the permeable solid (absolute permeability) and the multiple fluids (relative permeability and capillary pressure). These macroscopic descriptors are the volume-averaged continuum functions originated from the displacement mechanisms at pore scale (Bear, 1972). Therefore, the macroscopic transport of immiscible fluids can be predicted, if there are an adequate description of the essential geometry and topology of the pore space, and a sufficient representation of the multiphase flow physics. Nowadays, pore network models have become indispensable in the investigation of multiphase flow in porous media encountered in petroleum and environmental applications.

Numerical network models with various pore-space representations have been used to investigate many phenomena related to multiphase flow in porous media, including several recent papers investigating interfacial areas in multiphase flow. Lowry and Miller (1995) investigated the relationship between fluid saturation and fluid-fluid interfacial areas by using an irregular network structure of spherical nodes and cylindrical tubes. Reeves and Celia (1996) developed a network model consisting of spherical pores and constricted cylindrical tubes in a regular cubic network, and related interfacial areas to both capillary pressure and saturation. These various pore network models were reviewed by Blunt (2001). However most of these models are generated stochastically and usually do not represent natural permeable media. The recent advancements in rock imaging have resulted in a quantitative jump in the pore network model capabilities (Øren, et al., 1998; 2002; 2003; Patzek, 2001), and thus the Patzek model was used in this paper.

\section{Pore network model}

\subsection{Geometry description}

The pore network model used in this paper is a realistic representation of a sample of Berea sandstone reconstructed from the 3D micro-focused X-ray CT image, provided by Dr. Suicmez in Imperial College, London. Detailed description of reconstruction and its validation were discussed by Øren, et al. (1998; 2002; 2003) and Patzek (2001).

The network, with a spatial region of the volume of $3^{3} \mathrm{~mm}^{3}$, consists of 12,167 pores and 29,827 throats. 
Each pore or throat is represented by a duct with a triangular, square or circular cross-section, characterized by an inscribed radius which controls the threshold capillary entry pressure, effective corner angles which control the amount of fluid held in wetting layers, and an effective volume which controls the mobile (non-clay bound) saturation. In this model, 10 percent of these elements are squares, 5 percent are circular, and the rest are irregular triangles. The connection numbers varies from 1 to 12 , with an average of 4.8 . The porosity of the network is $36 \%$ and its absolute permeability is 2.5 Darcy.

\subsection{Pore and throat sizes}

The pore size distribution in this model follows the truncated Weibull distribution. And the inscribed pore radius can be described as follows:

$$
r=r_{\text {ch }}\left\{-\ln \left[(1-x) \mathrm{e}^{-\left(\frac{r_{\max }-r_{\min }}{r_{\text {ch }}}\right)^{\eta}}+x\right]\right\}^{\frac{1}{\eta}}+r_{\text {min }}
$$

where $x$ is a random number between 0 and $1 ; r_{\max }, r_{\text {min }}$ and $r_{\mathrm{ch}}$ are the maximum, minimum and characteristic inscribed radii, respectively, $\mu \mathrm{m}$; and $\eta$ is a dimensionless parameter. Here we set $r_{\max }=100 \mu \mathrm{m}$, $r_{\text {min }}=0.1 \mu \mathrm{m}$ and $\eta=1.5$.

The distribution of throat lengths also obeys the truncated Weibull distribution, and the minimum throat length is set at $20.0 \mu \mathrm{m}$ and the maximum throat length is $50.0 \mu \mathrm{m}$.

\subsection{Pore and throat shapes}

The shapes of pores and throats in the network are determined by shape factor, $G$. The shape factor is a dimensionless variable relating the cross-sectional area, $A$, of the element to its perimeter, $P$.

$$
G=\frac{A}{P^{2}}
$$

Square and circular elements have fixed shape factors of 0.0625 and 0.07958 , respectively. The shape factor of triangular elements obeys the truncated Weibull distribution, ranging from 0 (slit-shaped) to 0.04811 (equilateral).

\subsection{Capillary entry pressure}

Capillary entry pressure is the capillary pressure at which the non-wetting phase first displaces the wetting phase. In order for the NAPL to enter a pore, the differential pressure between the NAPL and the water must exceed the threshold capillary entry pressure of the pore. The capillary pressure is given by the
Young-Laplace equation:

$$
p=p_{\mathrm{NAPL}}-p_{\mathrm{W}}=\sigma_{\mathrm{NW}}\left(\frac{1}{r_{1}}+\frac{1}{r_{2}}\right)
$$

where $\sigma_{\mathrm{NW}}$ is the NAPL-water interfacial tension; and $r_{1}$ and $r_{2}$ are the principal radii of curvature of the interface. For a circular pore, Eq. (3) can be simplified as:

$$
p_{\mathrm{cNW}}=\frac{2 \sigma_{\mathrm{NW}}}{r} \cos \theta_{\mathrm{NWR}}
$$

where $\theta_{\mathrm{NWR}}$ is the receding oil-water contact angle; and $r$ is the inscribed radius (Dullien, 1992).

Eq. (4) shows that the smaller the pore spaces, the higher the capillary pressure. The minimum capillary entry pressure is the capillary pressure at which the non-wetting phase (NAPL) first displaces the wetting phase (water) contained in the largest pore throat within a water-wet pore network.

Only a very small fraction of network elements has a circular cross-section. For polygonal shaped elements the expression for the capillary entry pressure is complex as the wetting fluid remains in the corners as arc menisci. In this case, the capillary entry pressure can be calculated with the method proposed by Mayer and Stowe (1965)(Mason and Morrow, 1991) and Princen (1970), and this expression was generalized by Øren, et al. $(1998 ; 2002 ; 2003)$ :

$$
p_{\mathrm{c}}=\frac{\sigma \cos \theta_{\mathrm{R}}(1+2 \sqrt{\pi G})}{r} F_{\mathrm{d}}\left(\theta_{\mathrm{R}}, G, \beta\right)
$$

where $F_{\mathrm{d}}$ is a dimensionless correction factor for wetting fluids retained in the corners; and $\beta$ is the corner half angle of a triangular cross-section.

\section{Pore level displacement mechanisms and process}

There are two basic models of multiphase flow in pore networks: dynamic and quasi-static. In dynamic models, capillary, gravity, and viscous forces in the fluids are accounted for simultaneously. In quasi-static models, capillary force dominates; gravity modifies the order of magnitude of capillary pressure. In this study the quasi-static approach was used and the effect of viscous and buoyancy forces was ignored.

In the process of simulating two-phase flow in pore networks, the displacing fluid is injected through an external reservoir which is connected by pore throats to each pore body on the inlet side of the network. The displaced fluid escapes through the outlet face on the 
opposite side. Periodical boundary conditions are imposed along the sides parallel to the main direction of flow.

The displacing mechanism is critical for modeling microscopic water-NAPL displacement, therefore a suitable physical displacement should be adopted. In primary drainage, NAPL displaces water, the only invasion mechanism is piston-type displacement since initially the network is filled with water; whereas in secondary imbibition water displaces NAPL, part of the network has been invaded by NAPL, and the displacement mechanisms include piston-type, snap-off and cooperative pore-body filling. This classification of pore level events was first presented by Lenormand, et al. (1983), and the calculation of the corresponding capillary entry pressures was presented by Øren, et al. (1998; 2002; 2003) and Patzek (2001).

The step for primary drainage are summarized as follows: 1) Calculate all piston-type capillary entry pressures of links and arrange them in a strictly ascending order. 2) At each capillary pressure level, find all links that have capillary entry pressure less than the current level of capillary pressure, have not yet been invaded, and have at least one of the two attached nodes filled with NAPL. 3) Invade these links and the attached nodes if applicable. A node is ready for invasion if it is occupied by water and its piston-type capillary entry pressure is less than the current level of capillary pressure. 4) New links may now become available to invasion because the attached nodes have been invaded. 5) Repeat steps 2-3 until no further nodes or links can be invaded. 6) Having invaded all possible links and nodes, calculate the overall (average) water-phase saturation in the network. 7) Proceed to the next capillary entry pressure level and repeat steps 2-6 until the specified maximum capillary entry pressure is reached.

Similarly, the steps for secondary imbibition are: 1) Secondary imbibition starts after a maximum capillary pressure level in primary drainage has been reached. 2) Starting from the maximum capillary pressure level, invade links or nodes with water in the order of decreasing piston-type, and snap-off capillary entry pressures. All these pressures are sorted in a single list in a strictly descending order. 3). NAPL is displaced by the invading water in the alternating piston-type and snap-off manner, until the respective capillary entry pressure exceeds the current capillary pressure level. 4) Before proceeding to the next capillary pressure level, update the saturations. 5). Repeat step 3-5 at the next, lower capillary pressure level.

\section{Results and discussion}

\subsection{Effect of wettability}

The flow behavior in pore networks with different wettabilities, water-wet (advancing contact angles $20^{\circ}$ to $55^{\circ}$ ), intermediate-wet $\left(55^{\circ}\right.$ to $120^{\circ}$ ), and NAPL-wet $\left(120^{\circ}\right.$ to $\left.150^{\circ}\right)$, was compared. Fig. 1 shows that different wetting conditions result in different capillary pressures and relative permeabilities, thereby different two-phase flow behavior. Wettability has a significant effect on the capillary pressures and relative permeabilities. With the wettability of the pore network changing from water-wet to NAPL-wet, capillary pressures and NAPL relative permeability decrease gradually.

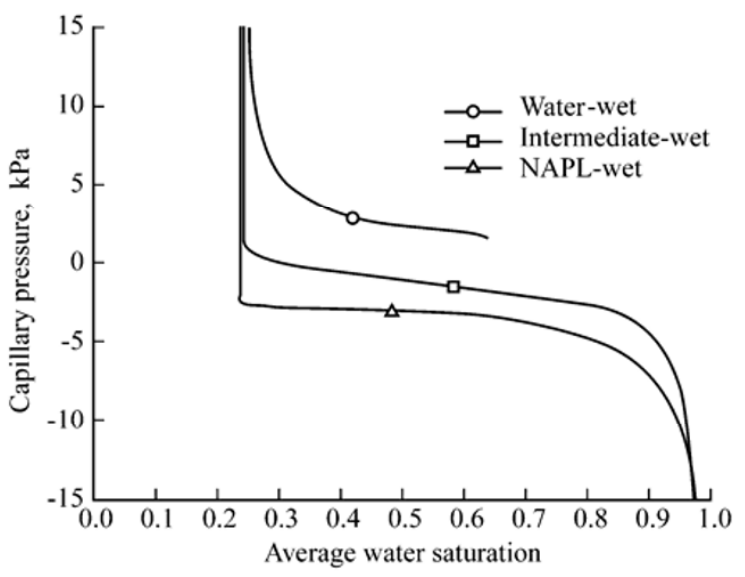

(a) Capillary pressure curves

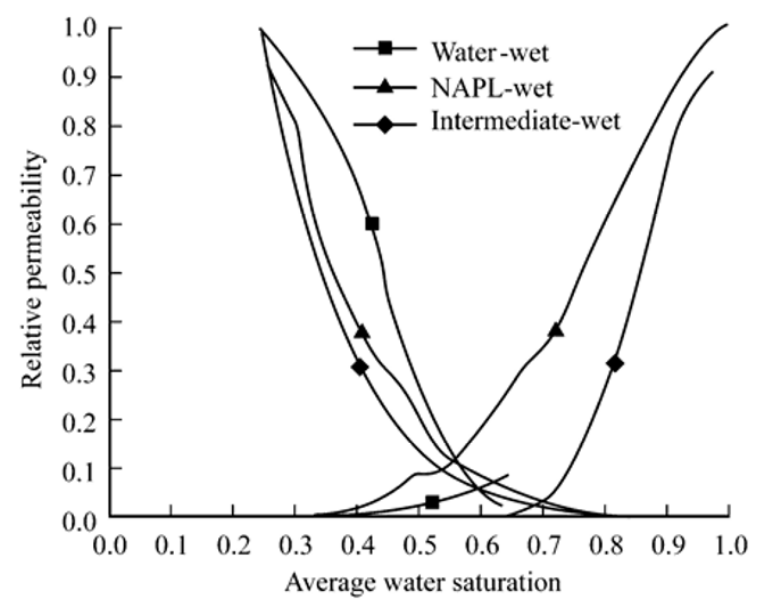

(b) Relative permeability curves

Fig. 1 Effect of wettability of pore network on capillary pressures and relative permeabilitis

In the case of NAPL-wet pore network, water relative permeability is the highest and NAPL relative permeability is the lowest. This is because NAPL 
moves in the thin intermediate layers, whereas water moves forward along the corners and the centers of the ducts. In the case of intermediate-wet network, water relative permeability is the lowest because water remains pinned near the duct corners without invading the duct centers. NAPL relative permeability is at first similar to that in the water-wet network, but then it becomes lower because of significant NAPL trapping. For the water-wet network, water relative permeability is between those in the intermediate-wet and NAPL-wet networks, while NAPL relative permeability is the highest because NAPL flows predominantly in the duct centers. It should be noted that near the trapped saturation, NAPL relative permeability curve has a positive slope for the water-wet network and approximately zero slope for other two networks.

The variability of two-phase flow in porous media can be explained by the competition of different pore-level displacement mechanisms in response to the change of advancing contact angle $\theta_{\mathrm{a}}$. In strongly water-wet systems, $\theta_{\mathrm{a}}=0$, link snap off is favored. As the capillary pressure decreases, snap-off fills the narrowest links with water throughout the network, and node filling is suppressed until most of the links are already filled. When most links are water-filled, NAPL in the nodes is likely to be trapped. Therefore, the domination of snap-off mechanism is responsible for the largest trapped NAPL saturation observed in the strongly water-wet systems. As we increase contact angle hysteresis, snap-off is favored less and piston-type displacement starts to dominate. The invasion starts by snap-off in the smallest links throughout the network, i.e., by ordinary percolation, followed by piston-like cooperative node filling if the node/link aspect ratio is not too large, i.e., by compact cluster growth (Lenormand, et al., 1983). The competition between different types of node filling mechanisms plays a major role in filling the nodes with water. The growth of compact water clusters leads to minor NAPL trapping, caused by bypassing or coalescence of the growing clusters of water-filled nodes and links. This observation indicates that in water-wet systems, the invasion of the water/NAPL central menisci present at the inlet face is favored less than snap-off in the network.

\subsection{Effect of the fraction of NAPL-wet pores}

The intermediate-wet network contains both water-wet and NAPL-wet ducts, the ratio of NAPL-wet ducts to water-wet ducts is important for secondary imbibition. Here the fraction of the NAPL-wet ducts, $f$, is defined as the ratio of the number of NAPL-wet ducts to the total number of ducts in the network. Fig. 2 shows capillary pressure and relative permeability as functions of the fraction of NAPL-wet ducts. It should be noted that slight changes in the fraction of NAPL-wet ducts result in drastic changes of flow characteristics in the pore network.

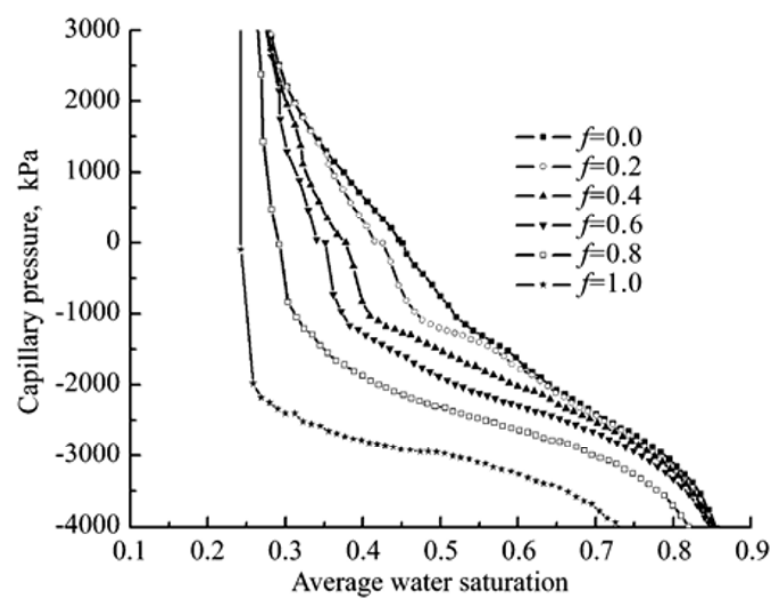

(a) Capillary pressure curves

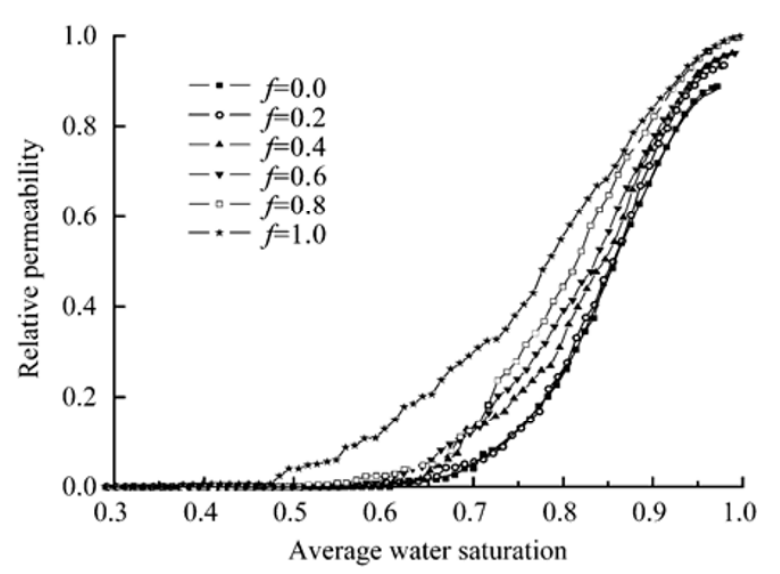

(b) Relative permeability curves

Fig. 2 Effect of fraction of NAPL-wet pores on capillary pressures and relative permeabilities

\subsection{Effect of NAPL/water interfacial tension}

For specified network wettability (i.e. contact angle), secondary imbibition proceeds on the water-wet, intermediate wet, and NAPL-wet networks at seven different values of interfacial tension. The capillary pressure curves are plotted in Fig. 3 for the water-wet, intermediate wet, and NAPL-wet networks, respectively. The relative permeability curve for the water-wet network is shown in Fig. 3d. 


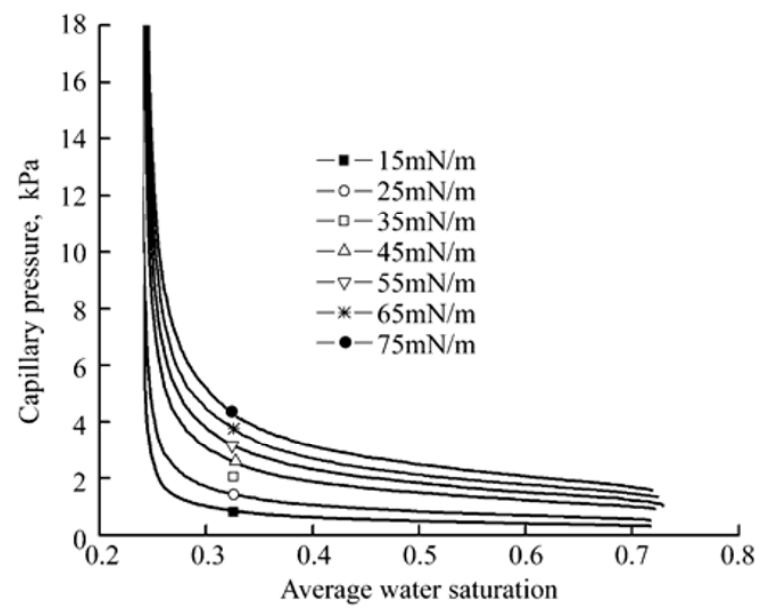

(a) Water-wet capillary pressure

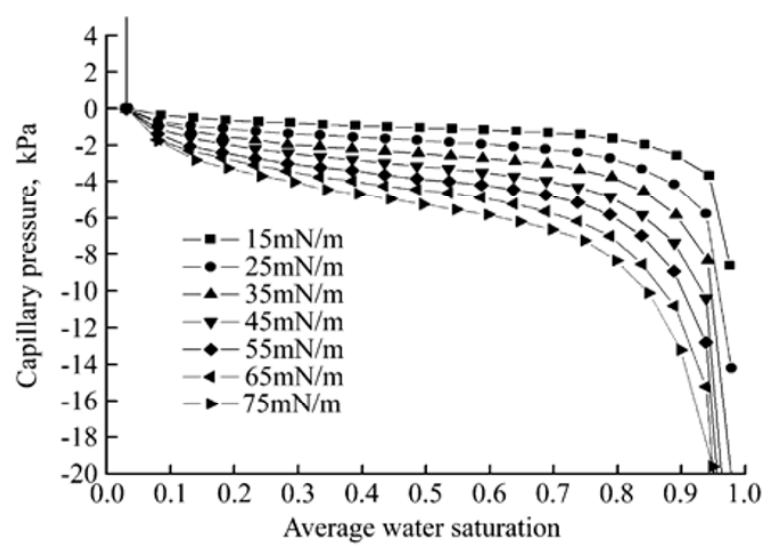

(c) NAPL-wet capillary pressure

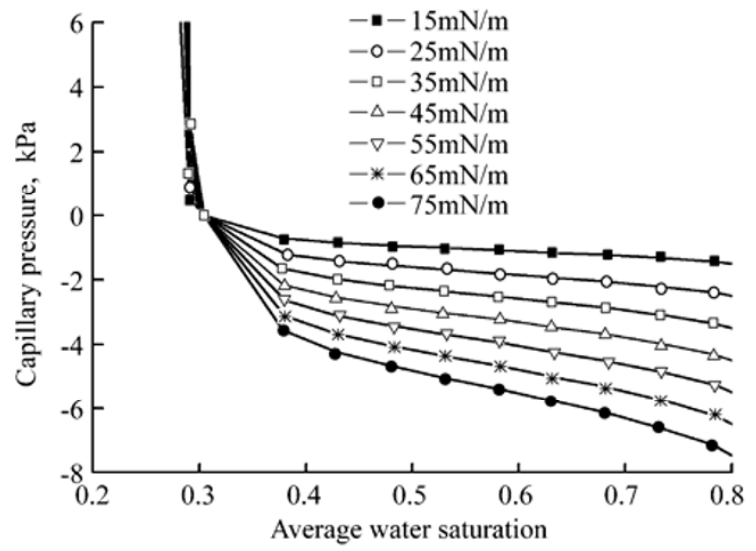

(b) Intermediate-wet capillary pressure

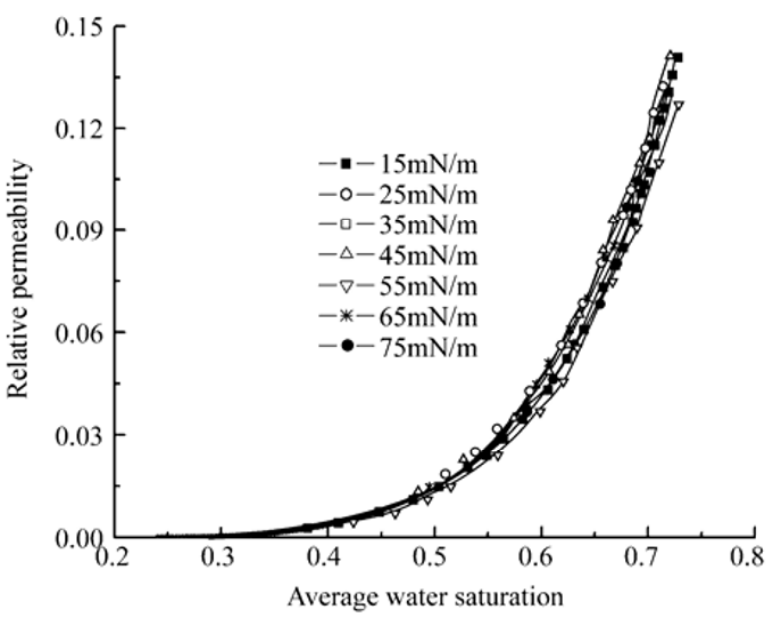

(d) Water-wet relative permeability

Fig. 3 Effect of interfacial tension on capillary pressures and relative permeabilities

The NAPL-wet secondary imbibition capillary pressure curves show more variability than the corresponding ones for the water-wet and intermediate-wet conditions. While NAPL/water interfacial tension has a slight effect on relative permeability.

\subsection{Effect of initial water saturation}

Fig. 4 shows initial water saturation, $S_{\text {wi }}$, has significant effect on capillary pressure and relative permeability. It should be noted that the pore capillary pressure is more significantly affected by NAPL-wet than water-wet or intermediate-wet network. For water-wet and intermediate-wet networks, the trapped NAPL saturation does not experience large changes as $S_{\mathrm{wi}}$ increases $( \pm 5 \%)$. However, in the NAPL-wet network, the trapped NAPL saturation increases noticeably with increasing $S_{\mathrm{wi}}$. Under each of the three wetting conditions, increasing $S_{\mathrm{wi}}$ decreases the water relative permeability, this result is certified by experimental data done by $\mathrm{Li}$ and his coworkers (2002).

For lower initial water saturation, most of the pores and throats are NAPL wetted, the water-NAPL displacing process is naturally of drainage. Under this condition, water occupies and moves along the large pores, and therefore high water relative permeability is observed. With an increase in initial water saturation, most large pores become water filled, and the small pores play more and more important role during displacement. Because of the small diameter, the tortuousness of pores increases and the water relative permeability decreases dramatically. So higher initial water saturation generates lower water relative permeability. 


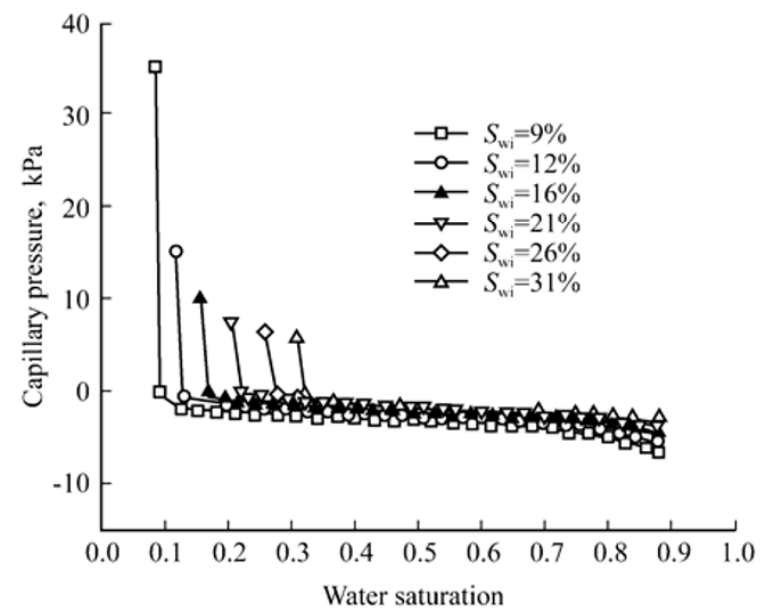

(a) Water-wet capillary pressure

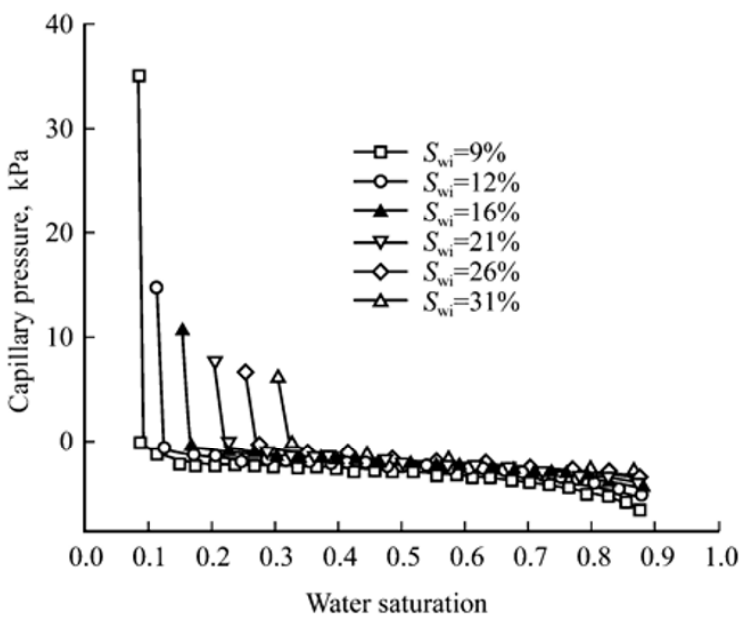

(c) NAPL-wet capillary pressure

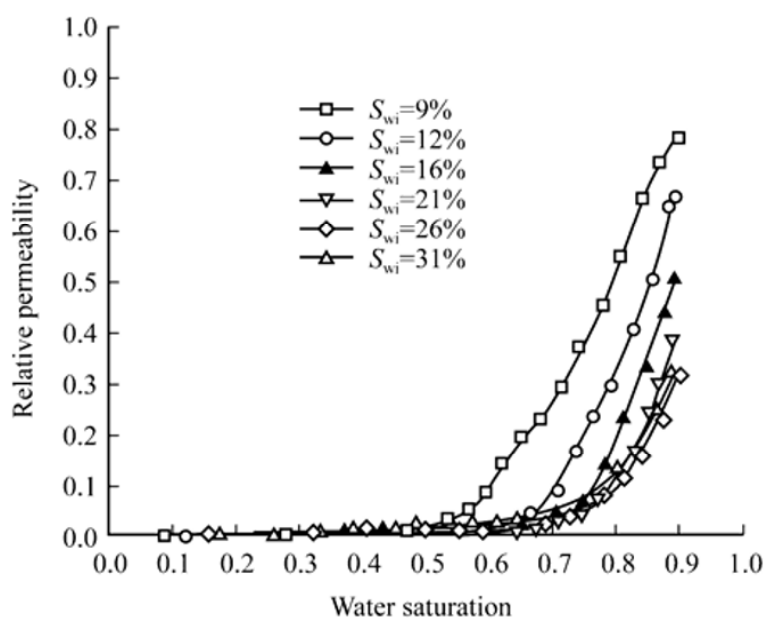

(e) Intermediate-wet relative permeability

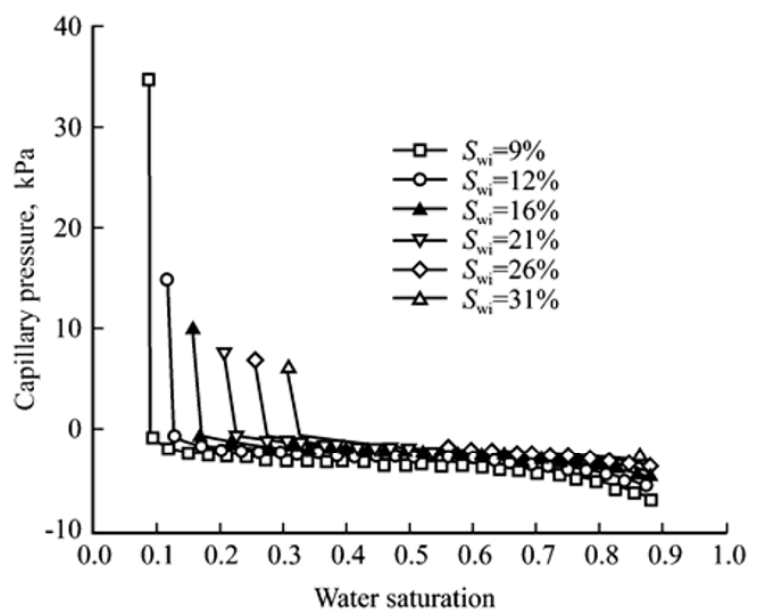

(b) Intermediate-wet capillary pressure

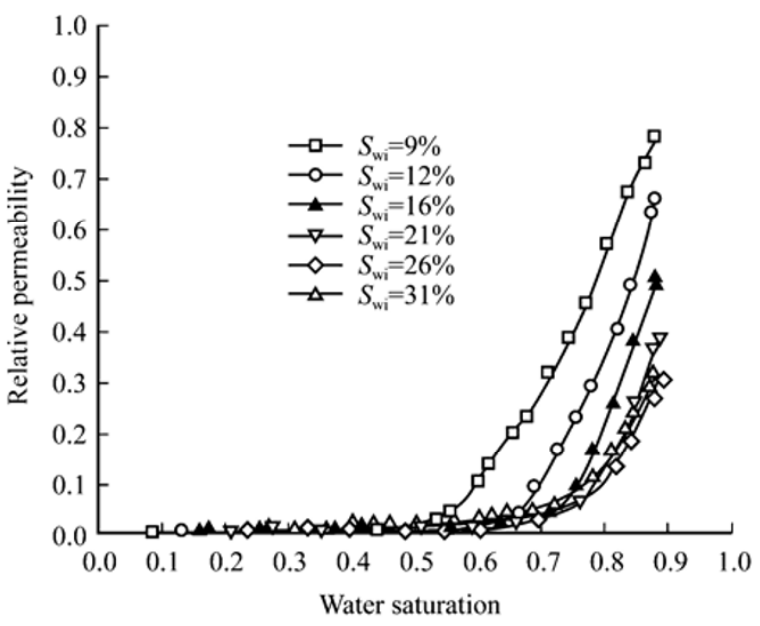

(d) Water-wet relative permeability

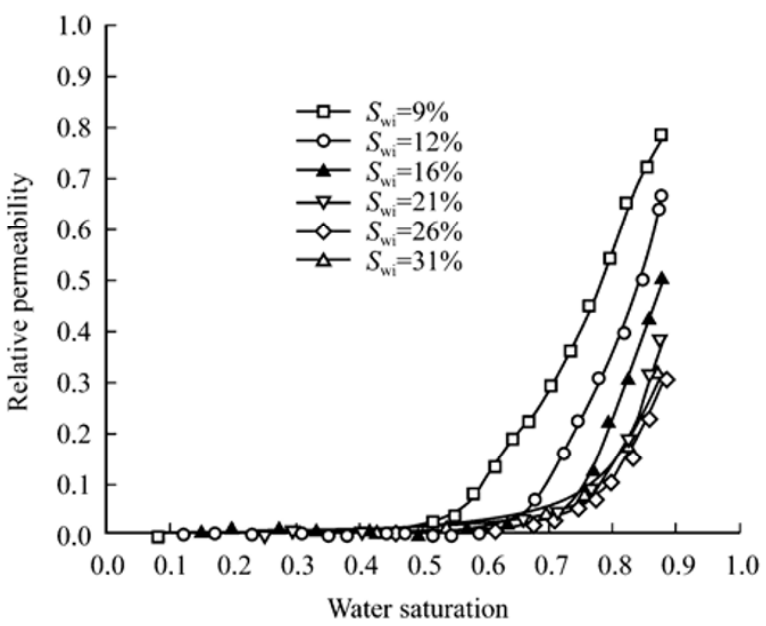

(f) NAPL-wet relative permeability

Fig. 4 Effect of initial water saturation on capillary pressures and relative permeabilities 


\section{Conclusion}

Wettability has a significant effect on NAPL-water displacement mechanism. For the water-wet pore network the displacement modes include snap-off, piston-like, and cooperative pore-body filling. For the intermediate-wet network, the flow is a mixture of spontaneous and forced secondary imbibition processes. For the NAPL-wet network, secondary imbibition is mainly forced piston-type displacement. The fraction of NAPL-wet pores strongly influences the two-phase flow behavior in the pore media.

\section{Acknowledgements}

This paper was supported by the National Natural Science Foundation of China (Grant No: 60473125).

\section{References}

Bear J. (1972) Dynamics of Fluids in Porous Media. American Elseviser Publishing Co., Inc.

Bedient P. B., Hanadi S. R., and Charles J. N. (1994). Groundwater Contamination: Transport, and Remediation, Prentice-hall. Englewood Cliffs, New Jersey.

Blunt M. J. (2001) Flow in porous media: Pore-network models and multiphase flow. Curr. Opin. Colloid Interface Sci., 6, 195-197

Dullien F. A. L. (1992) Porous Media: Fluid Transport and Pore Structure (2nd Edition). New York: Academic Press, 201-208

Li K. W., Chow K. and Horner R. N. (2002) Effect of initial water saturation on spontaneous water imbibition. SPE Western Regional/AAPG Pacific Section Joint Meeting held Anchorage, Alaska, 20-22 May 2002. SPE paper 76727

Lenormand R., Zarcone C. and Sarr A. (1983) Mechanisms of displacements of one fluid by another in a network of capillary ducts. J. Fluid. Mech., 16, 3365-3376

Lowry M. I. and Miller C. T. (1995) Pore-scale modeling of non wetting-phase residual in porous media. Water Resour. Res Res., 31(3), 455-473
Mason G. and Morrow N. R. (1991) Capillary behavior of a perfectly wetting liquid in irregular triangular tubes. $J$. Colloid Interface Sci., 141(1), 262-274

Mayer R. P. and Stowe R. A. (1965) Mercury porosimetry-breakthrough pressure for penetration between packed spheres. J. Colloid Interface Sci. 30, 893-911

Øren P. E., Bakke S. and Arntzen O. I. (1998) Extending predictive capabilities to network models. Soc. Pet. Eng. J. 3 (4), 324-336

Øren P. E. and Bakke S. (2002) Process based reconstruction of sandstones and prediction of transport properties. Transport Porous Media, 46, 311-343

Øren P. E. and Bakke S. (2003) Reconstruction of Berea sandstone and pore-scale modelling of wettability effects. Journal of Petroleum Science and Engineering, 39, 177-199

Patzek T. W. (2001) Verification of a complete pore network simulator of drainage and imbibition. Soc. Pet. Eng. J. 6, 144-156

Princen H. M. (1970) Capillary phenomena in assemblies of parallel cylinders: III Liquid columns between horizontal parallel cylinders. J. Colloid Interface Sci. 34, 171-184

Reeves P. C. and Celia M. A. (1996) A functional relationship between capillary pressure, saturation and interfacial area as revealed by a pore scale model. Water Resour. Res., 32(8), 2345-2358

\section{About the first author}

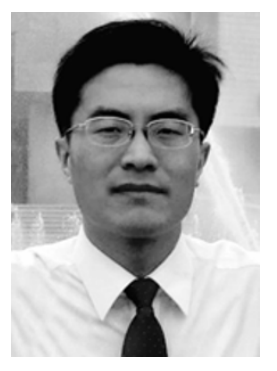

Yang Zhenqing was born in 1977 and received his MS degree from China University of Petroleum (Beijing) in 2006. Now he works in the Physics Department, China University of Petroleum (Beijing) with his major research interests in petroleum engineering and finite element analysis of rock mechanics.

E-mail: wireless@cup.edu.cn

(Received November 2, 2006) (Edited by Sun Yanhua) 